\title{
Case Report: E-cigarette or Vaping Induced Lung Injury
}

\section{Kyle Meggison*, Veronica Tucci and Alfredo Tirado}

Department of Emergency Medicine, HCA Healthcare/USF Morsani College of Medicine GME, Oak Hill Hospital, Brooksville, Florida

*Corresponding Author: Kyle Meggison, Department of Emergency Medicine, HCA Healthcare/USF Morsani College of Medicine GME, Oak Hill Hospital, Brooksville, Florida
Received: November 11, 2020

Published: December 22, 2020

(C) All rights are reserved by Kyle Meggison., et al.

\begin{abstract}
Electronic cigarettes have grown in popularity over the past few years. The medical literature and mainstream media have published accounts of e cigarettes causing severe respiratory distress in younger patients despite being marketed as a "safer" alternative to regular cigarettes. There have been cases throughout the country where individuals who use these products experience severe respiratory distress. This case report describes the clinical course of a young patient presenting to the emergency department in acute respiratory distress. We discuss the pathophysiology, incidence, prevalence, and morbidity and mortality of EVALI (E cigarette or vaping induced lung injury).
\end{abstract}

Keywords: Lung Injury; E-cigarette; Steroid Taper

\section{Case Report}

The patient was a 22-year-old female with no past medical history presenting to the emergency department with severe respiratory distress. She was tachycardia at 125 beats per minute, blood pressure $111 \backslash 59 \mathrm{mmHg}$, respirations 22 breaths per minute, oxygen saturation at $96 \%$ on a ventimask. Basic laboratory results were ordered including complete blood count (CBC), complete metabolic panel (CMP), arterial blood gas (ABG), Beta Human chorionic gonadotropin (bHCG). Chest xray and Cat scan angiography(CTA) of the chest were ordered. Patient states several hours before arrival she developed chest pain rated $9 \backslash 10$ that she described as substernal, tight, did not radiate, associated with progressive shortness of breath and unable to lay flat. Due to the rapid progression of her symptoms she called 911.

While laboratory investigations were pending a point of care ultrasound was performed which showed B lines suggesting an underlying pulmonary edema as seen in figure 1 .

Arterial blood gas showed 7.481/30.1/36.8/22.0/ on 5L nasal cannula (NC). CMP and CBC were within normal limits. Chest $\mathrm{x}$ ray was negative for any acute cardiopulmonary disease as seen in figure 2 .

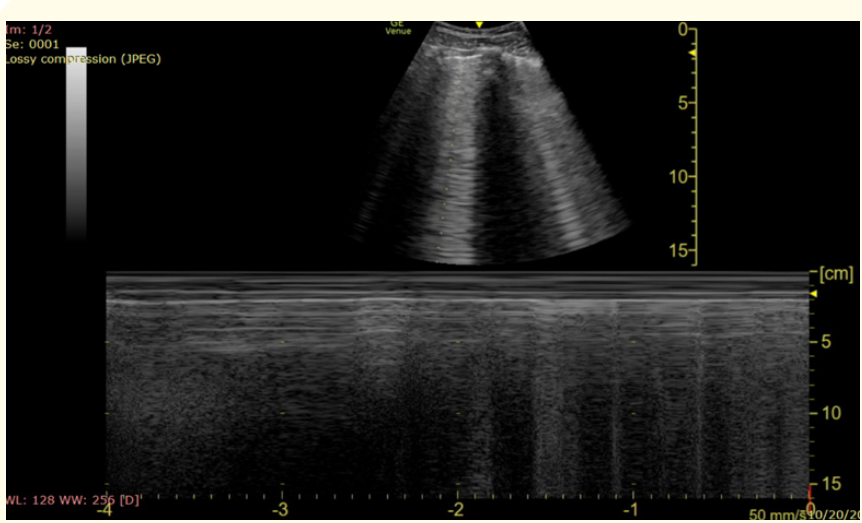

Figure 1: Point of care ultrasound showing B lines, indicating pulmonary edema.

CTA of the chest was ordered to rule out pulmonary embolism (PE), which showed nonspecific interstitial edema with apical basiliar gradient (see Figure 3). Bronchial wall thickening bilaterally, consistent with bronchitis, was also noted.

Patient was placed on Bilevel positive airway pressure (BiPAP) and had significant improvement in her respiratory sta- 


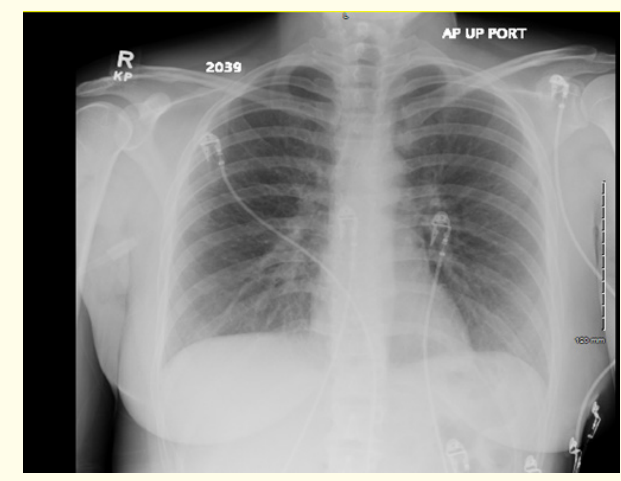

Figure 2: Upright AP chest $x$ ray.

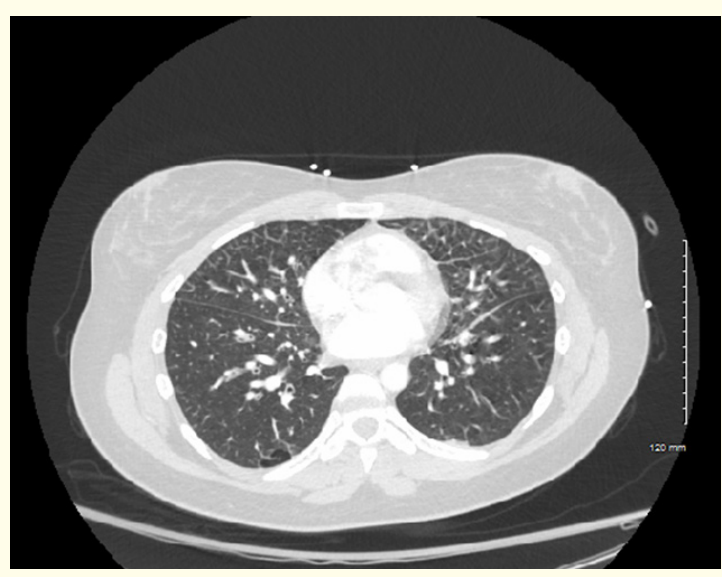

Figure 3: CTA chest.

tus. After discussing CTA results with the patient she revealed that she was a heavy E cigarette user. She was subsequently upgraded to the intensive care unit (ICU). Repeat ABG on BiPAP was 7.422/33.5/459.3/22/-1.5. In the ICU the patient was taken off Bipap in a few hours and started on azithromycin and Ceftriaxone by the ICU team. Patient was discharged home on the 5th day of admission with a steroid taper for one month.

\section{Discussion}

E-cigarettes are battery operated hand held devices that heat and atomize fluids for the user to inhale. This liquid generally contains nicotinic acid and may be flavored. The term vaping distinguishes e cigarette use from traditional tobacco products [1]. EVALI has swept the nation over the past year and at this time data is limited. Per the CDC we know that this condition disproportionately affects younger individuals with $80 \%$ of patients being age
$18-35$ and $66 \%$ of cases are men compared to women. A sociodemographic study done in Los Angeles County highlighted the relatively high levels (18.5\%) of unadjusted ever e-cigarette use among young adults (ages 18-24 years) in Los Angeles County compared to what has been found in other studies in the US (14.8\%). In Los Angeles County, age-adjusted prevalence of ever e-cigarette use was $11.1 \%$ among adult males and was $5.7 \%$ among adult females [2]. Our patient fits into this target demographic as she was a 22 y/o caucasian female.

In JAMA in 2019, an estimated $27.5 \%$ of high school students and $10.5 \%$ of middle school students reported current e-cigarette use. Among current e-cigarette users, an estimated 34.2\% of high school students and $18.0 \%$ of middle school students reported frequent use, and an estimated $63.6 \%$ of high school students and $65.4 \%$ of middle school students reported exclusive use of e-cigarettes. Among current e-cigarette users, an estimated $59.1 \%$ of high school students and $54.1 \%$ of middle school students reported JUUL as their usual e-cigarette brand in the past 30 days; among current e-cigarette users, $13.8 \%$ of high school students and $16.8 \%$ of middle school students reported not having a usual e-cigarette brand. Tobacco companies are also marketing to this age demographic using flavors. For example, with fruit, menthol or mint, and candy, desserts, or other sweets being the most commonly reported flavors with an estimated $72.2 \%$ of high school students and $59.2 \%$ of middle school students use flavored e-cigarettes [3].

A review of the literature shows that clinical symptoms are much like what was present in our case. Patients frequently complain of cough, shortness of breath, and chest pain. Other symptoms that have been reported are hemoptysis, fever, and chills none of which our patient had [4].

Clinically, these cases can appear as pneumonia or PE and as such these need to be excluded before a diagnosis of EVALI can be determined. In one study, $100 \%$ of patients had ground glass opacities on CT, which highly resembled acute respiratory distress syndrome [4]. Our patient however, had diffuse interstitial edema on CTA not ground glass opacities.

The pathophysiology at this time is still unclear. One study performed Bronchial alveolar lavage on affected patients and most had Vitamin E acetate or THC in the fluid. Nicotine was also detected in most samples and other items such as CBD, terpenes, petroleum distillates have been found in some samples [5]. At this time, it is unknown if one of these, a combination of, or possible metabolites 
are responsible for the lung damage seen in these patients. One study even suggested a possible production of ketene gas from vaping Vitamin E acetate that is toxic to lung tissue [6]. To this date, Vitamin $\mathrm{E}$ acetate is most strongly correlated with incidence of EVALI [7]. Our patient admitted to using nicotinic products and her urine drug screen was negative for marijuana or other substances.

The best treatment modality at this time is still unknown. In the emergency department, much of our treatment is based on patient's initial clinical presentation and history. EVALI is a diagnosis of exclusion and other etiologies should be ruled out before a diagnosis of EVALI is made. Other important considerations are pneumonia, PE, influenza, chronic obstructive pulmonary disease \ asthma exacerbation, and acute respiratory distress syndrome. Patients should be treated with supportive care. Antibiotics are recommended until an infectious process can be excluded and steroids are recommended only for patients who continue to deteriorate despite supportive care, although their efficacy is not understood at this time [4]. In our case, we initially treated the patient with supplemental oxygen and eventually upgraded to Bipap. Patient was continued on antibiotics during her stay and was discharged on a steroid taper.

The largest study performed to date shows that of those diagnosed with EVALI, 76\% required supplemental oxygen, 22\% required noninvasive ventilation, and $26 \%$ required intubation and mechanical ventilation. As of February $18^{\text {th }}$ of this year, 68 deaths have been reported out of 2807 hospitalizations per the CDC since March $31^{\text {st }}$ of $2019[8,9]$.

\section{Conclusion}

E-cigarette use has been a growing problem in the US. Although the rates of EVALI have decreased substantially since September of 2019, much of the long term risks of e-cigarette smoking is still unknown at this time, and further research will be needed to determine the risks, morbidity, and mortality associated with these products as well as appropriate standard therapy for treatment of those with EVALI.

\section{Bibliography}

1. National Academies of Sciences, Engineering, and Medicine; Health and Medicine Division; Board on Population Health and Public Health Practice; Committee on the Review of the Health Effects of Electronic Nicotine Delivery Systems; Eaton DL, Kwan LY, Stratton K, editors. Public Health Consequences of E-Cigarettes. Washington (DC): National Academies Press (US); E-Cigarette Devices, Uses, and Exposures (2018).
2. Levy DT., et al. "The prevalence and characteristics of E-cigarette users in the U.S". International Journal of Environmental Research and Public Health 10 (2017): 14.

3. Cullen KA., et al. "E-Cigarette Use Among Youth in the United States". JAMA 322.21 (2019): 2095-2103.

4. Layden JE., et al. "Pulmonary Illness Related to E-Cigarette Use in Illinois and Wisconsin - Final Report”. The New England Journal of Medicine 382 (2020): 903-916.

5. Blount BC., et al. "Vitamin E Acetate in Bronchoalveolar-Lavage Fluid Associated with EVALI". The New England Journal of Medicine 382.8 (2020): 697-705.

6. Wu D and O'Shea D. "Potential for Release of Pulmonary Toxic Ketene from Vaping Pyrolysis of Vitamin E Acetate". Proceedings of the National Academy of Sciences 117.12 (2020): 63496355.

7. Centers for Disease Control and Prevention. "Current cigarette smoking among adults in the United States” (2020).

8. Center for Disease Control and Prevention. "Outbreak of Lung Injury Associated with the Use of E-Cigarette, or Vaping, Products" (2019).

9. U.S. Food and Drug Administration. "Menthol and other Flavors in Tobacco Products" (2020).

\section{Assets from publication with us}

- Prompt Acknowledgement after receiving the article

- Thorough Double blinded peer review

- Rapid Publication

- Issue of Publication Certificate

- High visibility of your Published work

Website: www.actascientific.com/

Submit Article: www.actascientific.com/submission.php Email us: editor@actascientific.com Contact us: +919182824667 